ORIGINAL ARTICLE

\title{
$X$ chromosome influences sperm length in the stalk-eyed fly Cyrtodiopsis dalmanni
}

\author{
PM Johns and GS Wilkinson \\ Department of Biology, University of Maryland, College Park, MD, USA
}

\begin{abstract}
Whether sexually selected traits are sex linked can have profound effects on their evolution. In the diopsid stalk-eyed fly, Cyrtodiopsis dalmanni, sperm length and female reproductive tract morphology have coevolved across species, postcopulatory sexual selection is known to occur, and $\mathrm{X}$ linked genes affect female ventral sperm receptacle size. Here, we estimate the location of quantitative trait loci (QTL) for spermatocyst tail length by using F2 progeny segregating for an X-linked factor that causes sex-ratio meiotic drive. We found two QTL, including a major X-linked QTL responsible for
\end{abstract}

$25 \%$ of the variation in spermatocyst tail length $2.1 \mathrm{~cm}$ from the sex-ratio element and $0.8 \mathrm{~cm}$ from a major eye span QTL. Sexratio males produce shorter spermatocyst tails and shorter eye spans. Thus, X-linked factors affect both pre- and postcopulatory traits, and linkage between the alleles for short eye span and short spermatocyst tail length allow pre- and postcopulatory sexual selection to potentially act in concert against the transmission bias caused by the sex-ratio chromosome.

Heredity (2007) 99, 56-61; doi:10.1038/sj.hdy.6800963; published online 28 March 2007

Keywords: diopsidae; quantitative trait loci; sperm competition; sperm length; X-linked effects; sex-ratio

\section{Introduction}

Male and female reproductive morphologies have coevolved in several taxa (Pitnick et al., 1999; Presgraves et al., 1999; Minder et al., 2005; Beese et al., 2006). For example, in recent work on Drosophila melanogaster, Miller and Pitnick (2002) show that female seminal receptacle size influences male fertilization success and that lines selected for longer seminal receptacles evolve longer sperm. This correlated response to selection could occur either because of correlated selection acting on sperm or as a consequence of a genetic correlation between sperm and seminal receptacle sizes. Theory predicts such genetic correlations will arise whenever fertilizations occur nonrandomly as a consequence of a female preference for a male trait (Lande, 1981; Kirkpatrick, 1982). Coevolution of sperm size and female reproductive tracts can have remarkable outcomes: in some drosophila species, sperm is 20 times male body length, and sperm storage organ size in females is correspondingly exaggerated (Pitnick et al., 1999).

The degree to which sex-linked genes affect sexually selected traits can influence how the traits evolve. For example, sexual conflict can favor the initial increase of $\mathrm{X}$-linked recessive traits if they are beneficial to males but not to females (Rice, 1984; Charlesworth et al., 1987). Furthermore, the genetic correlation between ornament and choice traits depends on the mode of inheritance and the selection process (Hastings, 1994; Reeve and Pfennig, 2003; Kirkpatrick and Hall, 2004). For example, when both male and female traits are $X$ linked, sexual selection

Correspondence: Dr PM Johns, Department of Biology, Bard College, Annandale-on-Hudson, NY 12504, USA.

E-mail: philipjohns1@yahoo.com

Received 10 October 2006; revised 23 January 2007; accepted 16 February 2007; published online 28 March 2007 through a good genes process results in greater change in a female preference than when both traits are inherited on autosomes, whereas the reverse is true if sexual selection operates by a Fisher process (Kirkpatrick and Hall, 2004). Many sexually selected traits, including reproductive traits, are sex linked (Reinhold, 1998; Wolfenbarger and Wilkinson, 2001; but see Fitzpatrick, 2004). For example, X-linked genes are differentially expressed during spermatogenesis in mice (Wang et al, 2001) and humans (Torgerson and Singh, 2006). Similarly, sperm length is strongly influenced by X-linked genes in dung flies (Ward, 2000) and crickets (Morrow and Gage, 2001) although not in Drosophila mojavensis (Miller et al., 2003) or in hybrids produced from a cross between Drosophila simulans and Drosophila sechellia (Joly et al., 1997).

The evolution of sex-linked traits can also be profoundly influenced by the presence of meiotic drive. Meiotic drive is a selfish-gene phenomenon where drive alleles bias transmission in their own favor. When drive factors lie on the $\mathrm{X}$ chromosome, sex-ratio males bearing a driving (SR) $X$ chromosome typically sire extremely female-biased broods (Jaenike, 2001). Because SR/Y males pass few or no competent Y-bearing sperm (Peacock et al., 1972; Cazemajor et al., 2000; Wilkinson and Sanchez, 2001), the SR chromosome has a transmission advantage over a standard (ST) $X$ chromosome. Thus, in the absence of suppressors, SR can cause populations to become progressively female biased until selection opposes further change or the population goes extinct (Hamilton, 1967). In a female-biased population, females are under selection to avoid mating with males that carry drive chromosomes because females that mate with ST/Y males bear more sons and thus more grandchildren (Lande and Wilkinson, 1999). Thus, if ornaments indicate SR, then meiotic drive will accelerate sexual selection. 
The aim of this study was to map the chromosomal locations of quantitative trait loci (QTL) that influence sperm length in the stalk-eyed fly, Cyrtodiopsis dalmanni. Strong sexual selection operates both before and after mating in this species, and natural populations segregate for X chromosome meiotic drive (Presgraves et al., 1997; Wilkinson et al., 2003). Eye span in C. dalmanni is an elaborate, sexually dimorphic trait that males use to resolve contests (Panhuis and Wilkinson, 1999) and that females use as a basis for mate choice (Wilkinson and Reillo, 1994; Wilkinson et al., 1998). Eye span exhibits Xlinked inheritance (Wolfenbarger and Wilkinson, 2001) and eye span QTL are tightly linked to the sex-ratio element, presumably as a result of one or more inversions on the $X$ chromosome (Johns et al., 2005). As a consequence, SR males have shorter eye spans than ST males (Johns et al., 2005). Female C. dalmanni are also highly promiscuous (Wilkinson et al., 1998) thereby creating an opportunity for intense sperm competition or sperm selection (Corley et al., 2006; Wilkinson et al., 2006). Across species of diopsid stalk-eyed flies, male and female reproductive traits exhibit dramatic morphological diversity and evidence of correlated evolution (Presgraves et al., 1999), as might be expected if there is a sexual arms race fueled by postcopulatory sexual selection. Recent work revealed X-linked effects on female sperm storage organ size, but not sperm length (Wilkinson et al., 2005). However, the flies used in that study lacked drive $X$ chromosomes. Therefore, in this study we used flies that segregate for SR to determine whether the SR chromosome affects sperm length.

\section{Materials and methods}

\section{Experimental crosses}

To map QTL for sperm length we conducted an F2 intercross between lines of $C$. dalmanni selected for either long (high line) or short (low line) male eye span to body length ratio (relative eye span) for 45 generations (Wilkinson, 1993; Johns et al., 2005). We crossed a lowline SR/Y male to a high-line ST/ST female, which produced F1 females bearing a low-line SR X chromosome and a high-line ST $X$ chromosome, and F1 males with a high-line ST $X$ chromosome. We chose the parental male by screening 18 low-line males for sexratio and used the one that sired the most extreme offspring sex ratio (no sons and 95 daughters) to mate with a virgin high-line female. They produced six sons and more than 300 daughters. We then mated each of the six F1 males to a sister, allowed females to oviposit until death, and kept alive 310 male progeny from three families for at least 1 month before measuring spermatocysts and scoring them for evidence of meiotic drive (see below). We also collected females from the two largest families to use in creating a linkage map (see below).

\section{Phenotype measurement}

We measured the eye span, body length and thorax width of all F2 flies using $\times 11$ dissecting scope images at a resolution of 50 pixels $/ \mathrm{mm}$ using NIH Image, version 1.59 (http://rsb.info.nih.gov/nih-image/index.html). Meiotic drive alters spermatocyst head morphology in diopsid flies (Wilkinson and Sanchez, 2001). In
C. dalmanni, SR males produce sperm bundles with a characteristic 'singed hair' morphology because roughly half of the spermatocyst heads fail to elongate properly (Johns et al., 2005). Therefore, we used the length of the spermatocyst tail, that is, excluding the head, as our measure of sperm length. After teasing apart testes, we stained sperm bundles with $10^{-7} \mathrm{M}$ Hoechst 33258 (Sakaluk and O'day, 1984; Wilkinson and Sanchez, 2001), and then examined cysts at $\times 400(2.3 \mu \mathrm{m}$ per pixel) with ultraviolet fluorescence (Wilkinson and Sanchez, 2001). For each of 310 males from the three largest F2 families, we took two measurements of spermatocyst tails from each of four bundles of cysts beginning to disassociate to ensure development was complete.

\section{Genotyping}

We extracted genomic DNA from flies with Qiagen DNeasy kits (Qiagen, Valencia, CA, USA). After screening 52 microsatellite markers (Wright et al., 2004), we used 19 informative loci (Johns et al., 2005) to analyze the two largest families of 194 and 76 males and 271 and 197 females, respectively. We used fluorescently labeled primers in $5.5 \mu \mathrm{l} \mathrm{PCR}$ reactions (Wright et al., 2004) to amplify four X-linked and 15 autosomal loci. We separated amplification products by capillary electrophoresis using an ABI 3100 (Applied Biosystems, Foster City, CA, USA) and used GeneScan 3.1.2 (Applied Biosystems) and Genotyper 2.5 (Applied Biosystems) to assign genotypes to individuals.

\section{Linkage and QTL analysis}

We used the genotypes of the 468 F2 females and 270 F2 males to generate map distances and identify QTL for sperm length, as described in Johns et al. (2005). We assigned the 19 microsatellite marker loci and sex-ratio, as determined by spermatocyst bundle morphology, to three linkage groups and used Joinmap v. 3.0 (Van Ooijen and Voorrips, 2001) to generate map distances for each sex. We then used this linkage map when estimating QTL. As reported previously (Johns et al., 2005), all four X-linked markers co-segregated $2.1 \mathrm{cM}$ from the genetic element for SR as determined by spermatocyst morphology. The tight linkage means that one $X$ haplotype is almost perfectly associated with sex-ratio, and SR males have unique alleles at three of the four X-linked microsatellite loci. For this study, we used $X$ haplotye to identify males bearing SR or ST chromosomes.

We estimated QTL location and effect by composite interval mapping (Zeng, 1994) with MapManager QTXb20 (Manly et al., 2001). We performed 1000 genome-wide permutation tests to determine likelihood ratio (LR) critical values above which QTL were significant at $P<0.001$ (Doerge and Churchill, 1996), and used up to 2000 genome-wide permutation tests to estimate $P$-values for each QTL we found. We confirmed QTL location by recording the maximum LR obtained in each of 1000 bootstrap samples for each chromosome (Walling et al., 1998). When our analysis revealed more than one QTL, we included a background locus from that chromosome in the analysis. We used JMP v 5.0.1.2 (SAS Institute, Cary, NC, USA) to perform all other statistical analyses. 


\section{Results}

Spermatocyst tail length averaged $166.96 \pm 8.54$ (s.e.) $\mu \mathrm{m}$. We performed a hierarchical nested analysis of variance, treating as random effects F1 family, male within family and spermatocyst bundles within each male (Table 1). This analysis reveals that differences among males accounted for $42.6 \%$ of the measured variation in spermatocyst tail length, whereas differences among families accounted for only $6.4 \%$, and between duplicate measures accounts for only $0.5 \%$. We used the average spermatocyst tail length of each male for all further analyses. We found no effect of body length $(F=2.6$, $P>0.1$ ) on spermatocyst tail length. Variances across families were similar $\left(\mathrm{O}^{\prime}\right.$ Brien $\left.\mathrm{F}=0.72, P>0.4\right)$ and we used least squares estimates to correct for differences among families in spermatocyst tail length (LS sperm length). As in a previous study (Johns et al., 2005), we also used least squares to correct for family and body length effects on eye span (LS eye span).

When controlling for significant QTL on other chromosomes, our analysis revealed the location of two significant QTL for LS sperm length (Figure 1). One QTL (LR=14.1, $P=0.002)$ is located on the second chromosome and explains $4 \%$ of the additive variance in LS sperm length. The other QTL is located on the X chromosome ( $\mathrm{LR}=73.0$, $P=0.0005)$ and explains $25 \%$ of the additive variance in LS sperm length. Each QTL is located within $1 \mathrm{cM}$ of a microsatellite marker: ms-90 on the second chromosome, and the four co-segregating microsatellites $2.1 \mathrm{cM}$ from SR on the $X$ chromosome. We found no significant QTL for sperm length on the first chromosome.

Simple regression analysis revealed that LS eye span is a significant $(\mathrm{F}=24.1, P<0.0001)$ but weak $\left(r^{2}=0.08\right)$ predictor of LS sperm length. However, the relationship between LS eye span and LS sperm length disappears $(\mathrm{F}=0.77, P>0.3)$ when we include as factors the genotypes of the microsatellites closest to the QTL. The genotype at ms-90 $(\mathrm{F}=4.05, P=0.019)$ and the $X$ haplotype $(\mathrm{F}=98.1, P<0.0001)$ significantly affect LS sperm length. Males homozygous for the low-line genotype (LL in Figure 2) at the second chromosome QTL produce significantly longer sperm $(169.2 \pm 1.0 \mu \mathrm{m})$ than males homozygous for the high-line genotype $(\mathrm{HH}$ in Figure 2; 165.8 $\pm 0.71 \mu \mathrm{m}$; Tukey $\mathrm{HSD}=3.4$, where $Q=2.3$ at $\alpha=0.05)$. However, LS sperm length for neither homozygote is significantly different than that of the heterozygous genotype (HL in Figure 2). In contrast to the pattern at ms-90, males bearing the SR haplotype, which was inherited from the low-line parental male (L in Figure 2), produced significantly shorter LS sperm $(163.9 \pm 0.55 \mu \mathrm{m})$ than males bearing ST haplotypes $(170.8 \pm 0.44 \mu \mathrm{m} ; \mathrm{F}=95.0, P<0.0001)$, which were inherited from the high-line parental female ( $\mathrm{H}$ in Figure 2 ).

\section{Discussion}

Our main result, that a QTL explaining 25\% of the variation in sperm length is tightly linked to SR on the $X$ chromosome in C. dalmanni, is interesting in light of other

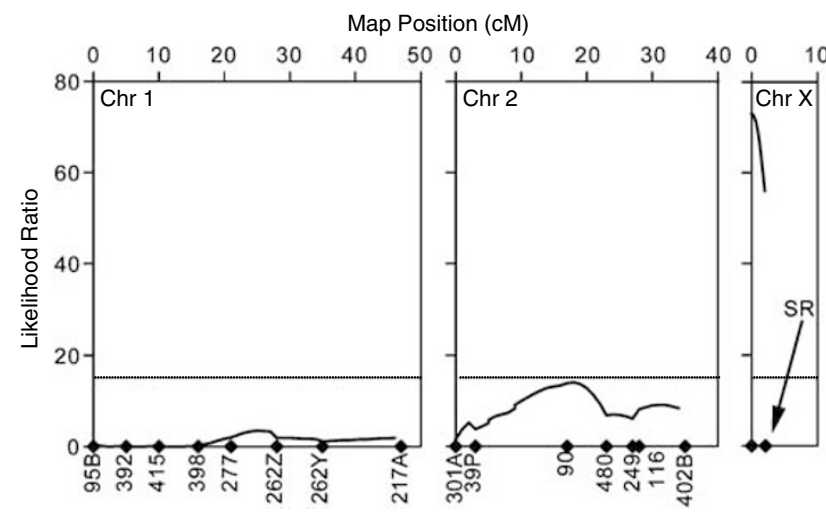

Figure 1 Quantitative trait loci (QTL) for LS sperm length, as estimated from F2 flies segregating for sex-ratio (SR). Chromosome (Chr) 1, 2 or $X$ indicated at the top of each panel. Microsatellite markers indicated by filled diamonds on $x$ axis below panels. Map positions in centimorgans (cM) indicated above each panel. LR indicated on the $y$ axis, where the dotted line indicates significance threshold for $P<0.001$. Notice that the four $X$-linked microsatellite loci used in this study (ms-54, ms-125, ms-244, and ms-395 (Wright et al., 2004)), co-segregate $2.1 \mathrm{~cm}$ from the SR genetic element (arrow).

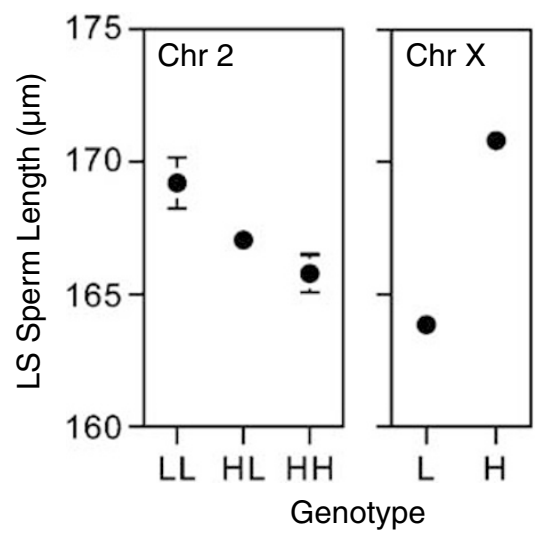

Figure 2 Mean $( \pm$ s.e.) LS sperm length $(\mu \mathrm{m})$ for F2 flies as a function of genotype for the microsatellite closest to QTL: ms-90 on chromosome 2 (Chr 2); and X haplotype (X). Source of alleles is given below each panel, where $\mathrm{L}$ and $\mathrm{H}$ refer to alleles inherited from the selected low and high lines, respectively (see text). The $\mathrm{X}$-linked low line allele is inherited from a sex-ratio (SR) $\mathrm{X}$ chromosome and the high line allele is inherited from a standard (ST) X chromosome.

Table 1 Nested ANOVA results for spermatocyst tail lengths of F2 offspring

\begin{tabular}{|c|c|c|c|c|c|}
\hline Source & d.f. & $M S$ & F-ratio & $\mathrm{P}$ & $\%$ of variance \\
\hline Family & 2 & 3566.5 & 10.7 & $<0.0001$ & 6.4 \\
\hline Male (family) & 309 & 332.3 & 4.4 & $<0.0001$ & 42.6 \\
\hline Bundle (family, male) & 932 & 76.4 & 220.9 & $<0.0001$ & 50.5 \\
\hline Residual & 1244 & 0.3 & & & 0.5 \\
\hline
\end{tabular}

Abbreviation: ANOVA, analysis of variance.

a Male nested within F1 Family. 
sex-linked effects in these flies. Johns et al. (2005) found an X-linked QTL that explained over one-third of the variation in eye span in the same cross. Recombination between the SR and ST X chromosomes is severely limited. In a control cross using flies derived from the same lines selected for long and short eye span, but lacking SR, the four X-linked microsatellites spanned $33 \mathrm{cM}$ (Johns et al., 2005). Meiotic drive systems are often associated with one or several inversions (Jaenike, 2001; Jutier et al., 2004). Inversions on the SR chromosome cause portions of the $\mathrm{X}$ chromosome to be protected from recombination and inherited together. Johns et al. (2005) found two X-linked QTLs in the control cross lacking SR. Thus, the single large X-linked eye span QTL in the experimental cross in that study represents the combined effects of at least two major genes. Similarly, the large Xlinked QTL for sperm length found in the current study could be a result of more than one $X$-linked gene.

The QTL for sperm length is tightly linked to the eye span QTL, which suggests that the X-linked genes affecting eye span and sperm length are bound in the same inversion complex. While we estimate only $0.8 \mathrm{cM}$ between the X-linked QTL for eye span and sperm length, the second chromosome QTL for sperm length maps $14.0 \mathrm{~cm}$ from the nearest QTL for eye span (Johns et al., 2005). Sex-ratio males, which inherited their $X$ chromosome from the parental low-line male, have both shorter spermatocyst tails and eye spans. However, the effect of the second chromosome QTL on sperm length is in the opposite direction to the QTL on eye span: selected high-line genotypes at the second chromosome are associated with longer eye span but shorter sperm length. Thus, whereas the X-linked genes that affect eye span and sperm length are inherited together when on an SR chromosome, the autosomal genes that affect eye span and sperm length are clearly independent.

The fly, C. dalmanni, is highly promiscuous (Wilkinson et al., 1998) thereby creating ample opportunity for intense postcopulatory sexual selection by sperm competition or selection. Comparative studies across stalkeyed flies reveal that sperm length, spermathecal duct length and ventral receptacle size have coevolved between males and females (Presgraves et al., 1999). The size and shape of female sperm storage organs have the potential to influence which sperm are used for fertilization. Thus, correlated evolution between male and female reproductive traits may be due, in part, to selection on sperm size mediated by the size of the female organs. However, correlated evolution between male and female reproductive traits may also be the result of linkage disequilibrium between pre- and postcopulatory traits. Among lines selected for long or short male eye span, Wilkinson et al. (2005) found a strong correlation between eye span and ventral receptacle size due, in part, to X-linked effects. Eye span is, therefore, partly indicative of both male and female postcopulatory features. Consequently, genetic linkage among X-linked genes that influence eye span, sperm length and ventral receptacle size provides a mechanism by which female mate choice can potentially influence male external morphology and sperm length as well as result in coevolution between sperm length and female sperm storage organ size. Furthermore, the genetic linkage between eye span and sperm length allows both pre- and postcopulatory sexual selection to act against the transmission bias caused by the sex-ratio chromosome.

The possibility that sperm length indicates SR males in postcopulatory competition, much as eye span indicates SR males in precopulatory mate choice, depends on how general the association between SR and sperm length is. The SR chromosome in our experimental cross has such a major effect on sperm length that it seems unlikely to be spurious. The X-linked QTL for sperm length explains nearly as much variation as the X-linked QTL for eye span. Although the parental SR male came from a line artificially selected for short eye span, and thus finding a major eye span QTL associated with SR is not surprising, there is no a priori reason to expect a QTL of similar magnitude for sperm length. However, without a control cross lacking SR, or a cross where the parental male has a different SR chromosome, we cannot determine whether the effect of SR on sperm length is specific to the particular SR haplotype present in the current cross or characteristic of all SR chromosomes. A recent study of C. dalmanni lacking SR found no evidence of X-linked effects on sperm length (Wilkinson et al., 2005), which suggests that the X-linked effects on sperm length reported here are specific to an SR chromosome. Because the SR chromosome rarely recombines (Wilkinson et al., 2006), it should evolve largely as a unit. Alleles for long sperm could, therefore, accumulate among recombining ST chromosomes throughout the population with little effect on the SR chromosome.

More than one SR chromosome exists in C. dalmanni populations (Wilkinson et al., 2006), and the effects of different SR chromosomes on sperm length may vary, but further crosses are needed to test this possibility. From this study alone, we cannot determine if the sperm length variation we observed is important for fertilization success in C. dalmanni. The difference of $6.9 \mu \mathrm{m}$ between average SR and ST LS sperm length in the current cross is more than $10 \%$ the cross-section of ventral sperm receptacles in unselected C. dalmanni Gombak females $(65.1 \pm 0.1 \mu \mathrm{m} ; \mathrm{EG}$ Amitin and GS Wilkinson, unpublished data) and about the same as the diameter of a sperm tubule $(6-8 \mu \mathrm{m}$, EG Amitin, personal communication), a magnitude large enough to suggest biological importance.

We know that sperm precedence in C. dalmanni is highly variable and exhibits a trimodal distribution (Corley et al., 2006). Furthermore, when females are double-mated to ST and SR males, ST males are twice as likely to fertilize offspring (Wilkinson et al., 2006). The greater sperm length of ST males may facilitate fertilization success. In other insect species, longer sperm do not necessarily have a fertilization advantage over shorter sperm (e.g., Gage and Morrow, 2003), but can in species where females mediate fertilization through organs like sperm receptacles (reviewed in Snook, 2005). We do not know the exact mechanisms of sperm competition in $C$. dalmanni; there may be a trade-off between producing many small or few long sperm, although very few sperm per ejaculate succeed in getting stored in female spermathecae (approximately 35 in Cyrtodiopsis whitei; Fry and Wilkinson, 2004). Male and female postcopulatory traits have coevolved across stalk-eyed fly taxa (Presgraves et al., 1999) and are the fastest evolving traits measured among populations within C. dalmanni (EG Amitin and GS Wilkinson, unpublished data), which are 
patterns consistent with sperm competition mediated by ventral receptacles. Double-mating experiments using flies that differ only in sperm length, but not presence of drive, are needed to determine how sperm length influences sperm competitive ability. By simultaneously allowing males to differ in eye span it may be possible to determine whether pre- and postcopulatory selection operates in concert or independently on male pre- and postcopulatory sexually selected traits.

\section{Acknowledgements}

We thank the people who helped with this study, especially Frances Lee, Sean Prager, Greg Brown, Mario Muscedere, Kasie Pratt, Jamie Walters and Tim Wright. This research was supported by an NIH postdoctoral traineeship to PMJ and by grants DEB-0077878 and DEB0343617 from the National Science Foundation to GSW.

\section{References}

Beese K, Beier K, Baur B (2006). Coevolution of male and female reproductive traits in a simultaneously hermaphroditic land snail. J Evol Biol 19: 410-418.

Cazemajor M, Joly D, Montchamp-Moreau C (2000). Sex-ratio meiotic drive in Drosophila simulans is related to equational nondisjunction of the $Y$ chromosome. Genetics 154: 229-236.

Charlesworth B, Coyne JA, Barton NH (1987). The relative rates of evolution of sex chromosomes and autosomes. Am Nat 130: 113-146.

Corley LS, Cotton S, McConnell E, Chapman T, Fowler K, Pomiankowski A (2006). Highly variable sperm precedence in the stalk-eyed fly, Teleopsis dalmanni. BMC Evol Biol 6: 53.

Doerge RW, Churchill GA (1996). Permutation tests for multiple loci affecting a quantitative character. Genetics 142: 285-294.

Fitzpatrick MJ (2004). Pleiotropy and the genomic location of sexually selected genes. Am Nat 163: 800-808.

Fry CL, Wilkinson GS (2004). Sperm survival in female stalkeyed flies depends on seminal fluid and meiotic drive. Evolution 58: 1622-1626.

Gage MJG, Morrow EH (2003). Experimental evidence for the evolution of numerous, tiny sperm via sperm competition. Curr Biol 13: 754-757.

Hamilton WD (1967). Extraordinary sex ratios. Science 156: 477-488

Hastings IM (1994). Manifestations of sexual selection may depend on the genetic basis of sex determination. Proc $R$ Soc Lond B Biol Sci 258: 83-87.

Jaenike J (2001). Sex chromosome meiotic drive. Annu Rev Ecol Syst 32: 25-49.

Johns PM, Wolfenbarger LL, Wilkinson GS (2005). Genetic linkage between a sexually selected trait and X chromosome meiotic drive. Proc $R$ Soc Lond B Biol Sci 272: 2097-2103.

Joly D, Bazin C, Zeng L-W, Singh RS (1997). Genetic basis of sperm and testis length differences and epistatic effect on hybrid inviability and sperm motility between Drosophila simulans and D. sechellia. Heredity 78: 354-362.

Jutier D, Derome N, Montchamp-Moreau C (2004). The sex-ratio trait and its evolution in Drosophila simulans: a comparative approach. Genetica 120: 87-99.

Kirkpatrick M (1982). Sexual selection and the evolution of female choice. Evolution 36: 1-12.

Kirkpatrick MK, Hall DW (2004). Sexual selection and sex linkage. Evolution 58: 683-691.

Lande R (1981). Models of speciation by sexual selection on polygenic traits. Proc Natl Acad Sci USA 78: 3721-3725.

Lande R, Wilkinson GS (1999). Models of sex-ratio meiotic drive and sexual selection in stalk-eyed flies. Genet Res 74: 245-253.
Manly K, Cudmore Jr RH, Meer J (2001). Map Manager QTX, cross platform software for genetic mapping. Mamm Genome 12: 930-932.

Miller GT, Pitnick S (2002). Sperm-female coevolution in Drosophila. Science 298: 1230-1233.

Miller GT, Starmer WT, Pitnick S (2003). Quantitative genetic analysis of among-population variation in sperm and female sperm-storage organ length in Drosophila mojavensis. Genet Res 81: 213-220.

Minder AM, Hosken DJ, Ward PI (2005). Co-evolution of male and female reproductive characters across the Scathophagidae (Diptera). J Evol Biol 18: 60-69.

Morrow EH, Gage MJG (2001). Artificial selection and heritability of sperm length in Gryllus bimaculatus. Heredity 87: 356-362.

Panhuis TM, Wilkinson GS (1999). Exaggerated male eye span influences contest outcome in stalk-eyed flies. Behav Ecol Sociobiol 46: 221-227.

Peacock WJ, Tokuyasu KT, Hardy RW (1972). Spermiogenesis and meiotic drive in Drosophila. In: Beatty RA, GluecksohnWaelsch S (eds). Spermiogenesis and Meiotic Drive in Drosophila. Bogtrykkeriet Forum: Copenhagen. pp: 247-268.

Pitnick S, Markow T, Spicer GS (1999). Evolution of multiple kinds of female sperm-storage organs in Drosophila. Evolution 53: 1804-1822.

Presgraves DC, Baker RH, Wilkinson GS (1999). Coevolution of sperm and female reproductive tract morphology in stalkeyed flies. Proc R Soc Lond B Biol Sci 266: 1041-1047.

Presgraves DC, Severence E, Wilkinson GS (1997). Sex chromosome meiotic drive in stalk-eyed flies. Genetics 147: 11691180.

Reeve HK, Pfennig DW (2003). Genetic biases for showy males: are some genetic systems especially conducive to sexual selection? Proc Natl Acad Sci USA 100: 1089-1095.

Reinhold K (1998). Sex linkage among genes controlling sexually selected traits. Behav Ecol Sociobiol 44: 1-7.

Rice WR (1984). Sex chromosomes and the evolution of sexual dimorphism. Evolution 38: 735-742.

Sakaluk SK, O'Day DH (1984). Hoechst staining and quantification of sperm in the spermatophore and spermathecae of the decorated cricket, Gryllodes supplicans (Orthoptera: Gryllidae). Can Entomol 116: 1585-1589.

Snook RR (2005). Sperm in competition: not playing by the numbers. Trends Ecol Evol 20: 46-53.

Torgerson DG, Singh RS (2006). Enhanced adaptive evolution of sperm-expressed genes on the mammalian $X$ chromosome. Heredity 96: 39-44.

Van Ooijen JW, Voorrips RE (2001). JoinMap 3.0 Software for the Calculation of Linkage Maps. Plant Research International: Wageningen, the Netherlands.

Walling GA, Visscher PM, Haley CS (1998). A comparison of bootstrap methods to construct confidence intervals in QTL mapping. Genet Res 71: 171-180.

Wang PJ, McCarrey JR, Yang F, Page DC (2001). An abundance of X-linked genes expressed in spermatogonia. Nat Genet 27: 422-426.

Ward PI (2000). Sperm length is heritable and sex-linked in the yellow dung fly (Scathophaga stercoraria). J Zool Lond 251: 349-353.

Wilkinson GS (1993). Artificial sexual selection alters allometry in the stalk-eyed fly Cyrtodiopsis dalmanni (Diptera: Diopsidae). Genet Res 62: 213-222.

Wilkinson GS, Amitin EG, Johns PM (2005). Sex-linked correlated responses in female reproductive traits to selection on male eye span in stalk-eyed flies. Integr Comp Biol 45: 500-510.

Wilkinson GS, Johns PM, Kelleher ES, Muscedere ML, Lorsong A (2006). Fitness effects of $X$ chromosome drive in the stalkeyed fly, Cyrtodiopsis dalmanni. J Evol Biol 19: 1851-1860.

Wilkinson GS, Kahler H, Baker RH (1998). Evolution of female mating preferences in stalk-eyed flies. Behav Ecol 9: 525-533. 
Wilkinson GS, Reillo PR (1994). Female preference response to artificial selection on an exaggerated male trait in a stalkeyed fly. Proc R Soc Lond B Biol Sci 255: 1-6.

Wilkinson GS, Sanchez MI (2001). Sperm development, age and sex chromosome meiotic drive in the stalk-eyed fly, Cyrtodiopsis whitei. Heredity 87: 17-24.

Wilkinson GS, Swallow JG, Christianson SJ, Madden K (2003). Phylogeography of sex ratio and multiple mating in stalkeyed flies from southeast Asia. Genetica 117: 37-46.
Wolfenbarger LL, Wilkinson GS (2001). Sex-linked expression of a sexually selected trait in the stalk-eyed fly, Cyrtodiopsis dalmanni. Evolution 55: 103-110.

Wright TF, Johns PM, Walters JP, Lerner AP, Swallow JG, Wilkinson GS (2004). Microsatellite variation among divergent populations of stalk-eyed flies, genus Cyrtodiopsis. Genet Res 84: 27-40.

Zeng Z (1994). Precision mapping of quantitative trait loci. Genetics 136: 1457-1468. 